\title{
Imaging genetics - towards discovery neuroscience
}

\author{
Tian $\mathrm{Ge}^{1,2}$, Gunter Schumann ${ }^{3}$ and Jianfeng Feng ${ }^{1,2, \star}$ \\ ${ }^{1}$ Centre for Computational Systems Biology, School of Mathematical Sciences, Fudan University, Shanghai 200433, China \\ 2 Department of Computer Science, University of Warwick, Coventry CV4 7AL, UK \\ ${ }^{3}$ Institute of Psychiatry, King's College London, London WC2R 2LS, UK \\ * Correspondence: jianfeng.feng@warwick.ac.uk
}

Received October 9, 2013; Revised December 4, 2013; Accepted December 5, 2013

\begin{abstract}
Imaging genetics is an emerging field aimed at identifying and characterizing genetic variants that influence measures derived from anatomical or functional brain images, which are in turn related to brain-related illnesses or fundamental cognitive, emotional and behavioral processes, and are affected by environmental factors. Here we review the recent evolution of statistical approaches and outstanding challenges in imaging genetics, with a focus on population-based imaging genetic association studies. We show the trend in imaging genetics from candidate approaches to pure discovery science, and from univariate to multivariate analyses. We also discuss future directions and prospects of imaging genetics for ultimately helping understand the genetic and environmental underpinnings of various neuropsychiatric disorders and turning basic science into clinical strategies.
\end{abstract}

Keywords: imaging genetics; association study; multiple testing; 5-O approach

\section{INTRODUCTION}

The past decade has witnessed a tremendous growth in brain imaging as well as an enormous explosion of interest and success in genomics. Imaging genetics, as an interdisciplinary field, aims to identify and characterize genetic variants that influence measures derived from anatomical or functional brain images, which are in turn related to brain-related illnesses or fundamental cognitive, emotional and behavioral processes, and are affected by environmental factors. An increasing number of studies have shown that many brain measures quantifying the organization or function of the human brain derived from complex image analyses are heritable and under genetic control, which indicates the first validity of analyzing the underlying genetic factors that influence these measures. Compared to many self-reported and questionnaire-based cognitive or clinical assessments in psychiatry, noninvasive brain imaging techniques provide much more objective and reproducible phenotypes, and can accommodate highly heterogeneous symptoms expressed by patients classified into the same diagnostic group. More importantly, quantitative brain imaging measures are considered to be intermediate or endophenotypes [1,2]
— on one hand, they are potential biomarkers (indicators of biological processes or treatment response) for and genetically related with particular mental disorders; on the other hand, they are closer to the disease process and have a simpler genetic architecture than disease syndrome and categorical diagnoses, and thus provide much greater power to identify and localize genetic loci [3]. In this context, brain imaging measures sit in the pathway that connects genetic factors to brain illnesses. Imaging genetics thus has the potential endpoint to help understand the genetic underpinnings of various neuropsychiatric disorders and improve early diagnosis and treatment. Moreover, brain images typically have high spatial resolution, and thus offer the opportunity to precisely localize genetic influences or map the spatial profile of the genetic effects over the entire brain. Partly due to these reasons, public and private funding resources have poured their investments into the field of imaging genetics with the hope of boosting the studies in this area, and ultimately to improve human health care in the future [4].

Although standard imaging-only or genetic-only methods are well established and have many successful applications, merging the two fields is not straightforward. To handle the extremely high dimensional imaging 
and genetic data set with complex covariance structures, joint efforts are needed from the communities of mathematicians, statisticians and computer scientists, along with the guidance from geneticists, neuroscientists, psychologists and clinicians. In this review article, we summarize our current knowledge on recent methodological developments and innovations in imaging genetics. We start with a brief introduction to quantitative neuroimaging phenotypes and the molecular basis of imaging genetic studies. We then focus on populationbased imaging genetic association studies and show the evolution of imaging genetics from candidate approaches to whole-brain and genome-wide searches, and from univariate analyses to multivariate analyses. We close the review with a discussion on the prospects and future directions of imaging genetics.

\section{Neuroimaging phenotypes}

In principle, any measures extracted and derived from different brain imaging modalities can be used as phenotypes in imaging genetic studies. These typically involve voxel-, vertex-, surface- or connection-based traits computable from structural, functional or diffusion images. For example, the volume or concentration of gray or white matter in a particular brain region can be extracted from structural images, using methods such as voxel-based morphometry [5]. Cortical thickness refers to the distance between the pia mater and the gray-white matter interface, and captures the variability of the six cortical layers. Cortical area refers to the area of the pia mater and the gray-white matter interface. These surfacebased measures of a particular brain region can be computed using surface representations and computational models of the cerebral cortex [6-8]. A number of studies have shown that these features of cortical morphology are heritable and regionally influenced by distinct genetic factors $[9,10]$. Other morphological measures, such as gyrification index (the ratio between the pial surface and its convex hull), quantify the complexity and folding patterns of the cerebral cortex. They have yet been widely investigated in the imaging genetic context, and deserve future studies. Brain imaging phenotypes can also be extracted from functional MRI images. The extent of activation or deactivation of brain areas in response to external stimuli can be estimated from functional brain images. The level of spontaneous brain activity under resting state also has implications on function of the brain and may be used as imaging traits $[11,12]$. Recently, the field of connectome genetics, which investigates how genetic factors affect both anatomical and functional brain connectivity, is rapidly expanding [13]. In particular, diffusion tensor imaging (DTI) provides a number of measures, such as fractional anisotropy (FA), mean diffusivity (MD), radial diffusivity (RD), and axial diffusivity (AD), which reflect the coherent direction of axons and assess white matter integrity. These measures are highly heritable $[14,15]$ and related with a variety of neuropsychiatric disorders [16]. More complex variants of DTI, such as diffusion spectrum imaging (DSI) and high angular resolution diffusion imaging (HARDI), are more sensitive to intravoxel heterogeneities in diffusion directions caused by crossing fiber tracts, and thus provide more accurate mapping of anisotropy. Tractography calculates the likelihood for tracts and reconstructs fiber tracts using diffusion and anisotropy [17]. Thus it largely reflects the physical connection between brain regions and can be used as imaging phenotypes as well. Functional connectivity between brain regions, assessed from task based or intrinsic brain activities, also provides heritable and sensitive measures of functional integration and information processing in the brain [18-20]. Finally, the totality of anatomical and functional connectivities in the brain can be viewed as networks and represented as graphs. Many topological graph theory measures [21] can then be used to evaluate the local and global organization of the structural and functional connections in the brain. Table 1 summarizes different types of neuroimaging phenotypes.

Although brain images provide numerous candidate phenotypes for imaging genetic studies, selected imaging traits should be highly heritable and be genetically related with the mental disorder or biological process under investigation as closely as possible. Some imaging traits may be less relevant to the process of interest, less reproducible in a particular experimental design or data collection protocol, or simply noisier than other measures, and thus have reduced statistical power to localize genetic variants. However, there are currently few statistically rigorous methods for optimally choosing appropriate

Table 1. Summary of neuroimaging phenotypes

\begin{tabular}{lll}
\hline Morphology & Function & Connectome \\
\hline $\begin{array}{l}\text { Volume/concentration of gray/white matter; } \\
\text { cortical thickness; cortical area; gyrification } \\
\text { index }\end{array}$ & $\begin{array}{l}\text { Extent of activation/deactivation; } \\
\text { contrast map; measures characterizing } \\
\text { spontaneous brain activity }\end{array}$ & $\begin{array}{l}\text { Anisotropy (FA, MD, RD, AD); tractography; } \\
\text { functional/effective connectivity measures } \\
\text { (correlation, coherence, causal influences, etc.); } \\
\text { complex network measures }\end{array}$ \\
\hline
\end{tabular}


endophenotypes given the complexity and high dimensional nature of brain imaging data, and the largely unknown biological mechanisms that underlie mental illnesses.

Winkler et al. [10] examined the relationship between brain volume, grey matter volume, cortical thickness and surface area from a genetic standpoint. These measures were extracted using both surface-based and voxel-based methods, and their heritability and genetic correlations were estimated from 468 individuals with extended pedigrees design using a variance-component model. They confirmed that cortical thickness and surface area measures were genetically and phenotypically independent, suggesting that surface area and cortical thickness measures should be considered separately in imaging genetic studies. Moreover, they found that, although all imaging traits were significantly influenced by genetic factors, compared to voxel-based grey matter volumes which have mixed genetic components, surface-based measures such as surface area and cortical thickness should be preferred for imaging genetic studies.

Glahn et al. [22] proposed a novel objective index of the genetic utility of endophenotypes for an illness, termed as the endophenotype ranking value (ERV), which is able to choose appropriate endophenotypes that are heritable and have high level of pleiotropy with the illness of interest. They applied the ERV analysis to a high dimensional set of over 11000 traits drawn from behavioral/neuro-cognitive, neuro-anatomic, and transcriptomic phenotypic domains, and identified a set of top-ranked endophenotypes for recurrent major depression in a sample of 1122 individuals from large randomly selected extended pedigrees. This efficient screening method has the potential to prioritize more appropriate endophenotypes for imaging genetic analyses.

To summarize, although brain imaging techniques provide an array of potential endophenotypes, computationally efficient and statistically rigorous methods are needed to facilitate the identification of endophenotypes that are most appropriate for imaging genetic studies. "Fishing" and vibration, i.e., screening measures and adjusting analysis methods until some promising findings appear, should be avoided.

\section{Genetic background}

DNA is an informational molecule containing the genetic information used in the development and functioning in all living organisms. It is a macromolecule that is essential for all known forms of life. In humans, DNA molecules are double-stranded helices, consisting of two long polymers made of about 3 billion nucleotides. Each nucleotide has its backbone made of a five-carbon sugar (deoxyribose) and a phosphate group, and a nucleobase attached to the sugar. There are four types of nucleobase - adenine (A), thymine (T), guanine (G) and cytosine (C). The two strands of DNA run in opposite directions to each other and are complementary, i.e., adenine bonds only to thymine and cytosine bonds only to guanine. It is the sequence of these nucleobases along the backbone that encodes information. Within cells, DNA is highly compressed and organized into long structures called chromosomes. Humans have 23 pairs of chromosomes - 22 pairs of autosomal chromosomes and one pair of sex chromosomes.

A gene is a molecular unit of heredity in a living organism. It is a DNA segment that contains information to make proteins. Therefore, genes hold the information to build and maintain an organism's cells and pass genetic traits to offsprings. Not all DNA sequences encode proteins. There are regions called introns that are removed after DNA is transcribed into ribonucleic acids (RNA). Only the exons are transcribed into messenger RNA, which is later translated into proteins. Humans have about 20000 to 25000 genes and their coding regions compose about only $1 \%-2 \%$ of our genome. The function of $98 \%$ of our DNA is largely unknown.

Imaging genetics assesses the variation in brain imaging phenotypes that can be explained by genetic variation. Genetic variation can be caused by mutations or during chromosomal events like cell division and sexual reproduction. It provides the genetic material for natural selection. Genetic variation may occur at a variety of levels - the genome level, the chromosome level, the gene level, and at single nucleotides. Many of the variations are copy-number variations (CNVs), which refer to an abnormal number of copies of one or more sections of the DNA. CNV is a form of structural variation that corresponds to relatively large regions of the genome that have been deleted or duplicated, and may be tested for association with imaging traits.

In contrast to CVNs, a majority of the current imaging genetic studies focus on a particular type of genetic variation, the single nucleotide polymorphism (SNP), which is a DNA sequence variation at a single nucleotide. Each alternative form at a single nucleotide is called an allele. Almost all common SNPs have only two alleles. Therefore, the more frequent allele in the population is called the major allele and the rarer the minor allele. The lower allele frequency at a locus that is observed in a particular population is called the minor allele frequency (MAF). Since humans are diploid (i.e., there are two physical copies of each chromosome in the cell), each SNP can be coded in the form of 0 (homozygotic major alleles), 1 (heterozygote) and 2 (homozygotic minor alleles). There are more than 20 million common SNPs and many more rare SNPs (MAFs $<1 \%-5 \%$ ), and their genomic distribution is not homogenous. SNPs may fall 
within coding sequences of genes, non-coding regions of genes, or in the intergenic regions. Even though it lies in the exonic region of a protein-coding gene, a SNP does not necessarily change the amino acid sequence of the protein that is produced (synonymous polymorphism) due to the redundancy of the genetic coding. However it may also be a missense polymorphism (resulting in a different amino acid) or a nonsense polymorphism (resulting in a premature stop). Moreover, SNPs that are not in proteincoding regions may still affect gene splicing, transcription factor binding, messenger RNA degradation, or the sequence of non-coding RNA.

A key concept in association studies is linkage disequilibrium (LD), which refers to non-random association of alleles at two or more loci. This means that certain pairs of alleles in a population appear more often (or less often) than expected by chance based on their frequencies. LD is due to the physical connection between nearby loci on a chromosome, which is known as genetic linkage, referring to the tendency of two alleles proximal to each other to be inherited together from generation to generation. LD can be quantified by the difference between observed and expected allelic frequencies, but the level of LD is influenced by a number of factors and is largely unpredictable. One factor that causes the decay of LD is genetic recombination, or more specifically, the chromosomal crossover occurring during meiosis - a special type of cell division necessary for sexual reproduction. Homologous chromosomes exchange sections or fragments of their genetic materials, which produces chromosomes with a new combination of genes. This crossover of DNA can cause alleles previously on the same chromosome to be separated and end up in different daughter cells, and hence break the LD. Since, in general, nearby alleles tend to be inherited together and distant alleles have a higher probability of recombination, it is expected that alleles proximal to each other are in high LD while alleles with a large physical distance are almost uncorrelated. A combination of alleles at adjacent loci on the chromosome that are transmitted together (and therefore statistically associated) is called a haplotype block [23]. However, since the recombination rate is highly inhomogeneous over the genome, the extent of LD or the size of a haplotype block varies between a few kilobases and a few hundred kilobases long in humans. One useful aspect of LD is that it reduces the number of SNPs necessary in whole-genome SNP association studies. For a region of the genome with high LD, only a single representative SNP is needed to capture most of the genetic variability in the region. These highly informative SNPs are called tag SNPs. It is known that $99 \%$ of the approximately 20 million common SNPs (with MAFs $>5 \%$ ) can be reasonably tagged by only one million tag SNPs.

\section{Population-based association studies}

Association is one of the main ways to localize chromosomal regions that influence a trait. Genetic association study tests if a genetic variation helps to predict the phenotype. In contrast to another gene discovery method, linkage analysis, which requires a twin or pedigrees design, association analysis can be conducted on unrelated individuals, making it easier to recruit a relatively large number of subjects. Due to the efficient and straightforward statistical methods, association study is now a common and practical approach for gene discovery. However, association analysis also suffers from several major issues and caution is needed in interpreting the findings.

Currently, association studies are exclusively focused on a subset of common genetic variants, which do not capture the entirety of the genetic variation. As a result, association analysis relies on the LD among nearby genetic variants, and works only when (1) The causative SNP (the SNP that is mechanically related to the phenotype) is genotyped, or (2) The genotyped marker is in disequilibrium with a causative SNP. Since LD is largely unpredictable and the sizes of haplotype blocks vary across both the genome and populations, the use of LD makes it challenging to estimate statistical power for genetic association studies. One needs to be careful that a positive finding is not necessarily a causative SNP but may be in LD with an approximal causative SNP, while negative results have implications only for the marker tested and do not exclude the entire gene or genetic region. Association studies typically provide a localized chromosomal region that is approximately 500 kilobases that harbors the causative SNP. Follow-up analyses are required to identify the causal variants and study their biological effects. The situation may change with the recently available whole genome sequence data that genotypes all the 3 billion nucleotides in the genome and captures the totality of human genetic variation.

Population stratification is another potential source of bias in association studies. If a sample collected for genetic association analysis contains multiple populations that systematically differ in the trait of interest, any locus whose allele frequencies differ between the populations could show an erroneous association $[24,25]$. Therefore, the genetic origins of the samples included in association analyses should be kept as homogeneous as possible. Statistical corrections for population stratification, such as genomic control [26], EIGENSTRAT [27] and EMMAX [28], are well developed and largely successful.

We now review recent methodological developments for population-based imaging genetic association studies, in an order that reflects the trend of imaging genetic studies - from candidate approaches to whole-brain 
genome-wide searches, and from univariate analyses to multivariate analyses. We summarize some merits and disadvantages of the methods reviewed in the paper in Table 2.

\section{CADIDATE-GENE CADIDATE- PHENOTYPE ANALYSES}

The field of imaging genetics started with candidate-gene and candidate-phenotype studies, as it was uncommon, and costly, to scan and genotype a large number of subjects to obtain both whole-brain and genome-wide data. Prior biological knowledge is needed to select a handful of specific, well-studied genetic variants and a few characteristic measures of brain anatomy, function, or connectivity that are under genetic control and are intermediate phenotypes of the biological process of interest. Candidate approaches allow people to test biologically plausible hypotheses and determine how specific genetic variants affect brain structure and function in a range of neurological and psychiatric disorders. Strong hypotheses often drive clearly interpretable results.
For example, attention-deficit/hyperactivity disorder (ADHD) symptoms include impulsivity and hyperactivity, which are associated with the activation of the reward area of ventral striatum (VS) and the principal inhibitory frontal area, the right inferior frontal gyrus (IFG), during reward anticipation and response inhibition, respectively. Nymberg et al. [29] investigated how these fronto-striatal activation patterns contribute to ADHD symptoms depending on the genotype of SNP rs12843268 on monoamine oxidase $\mathrm{A}(M A O A)$ gene, which is localized on the human $\mathrm{X}$ chromosome and thought to underlie neural functions associated with ADHD. They found, in a sample of adolescent boys, that in A hemizygotes of the SNP, ADHD symptoms are associated with lower VS blood-oxygen level dependent (BOLD) response during the monetary incentive delay (MID) task and lower IFG BOLD response during the stop signal task (SST). In G hemizygotes, ADHD symptoms are associated with increased IFG BOLD response during the SST in the presence of increased VS BOLD response during the MID task (Figure 1). Thus, ADHD symptoms may be associated with reward deficiency or insufficient response inhibition.
A

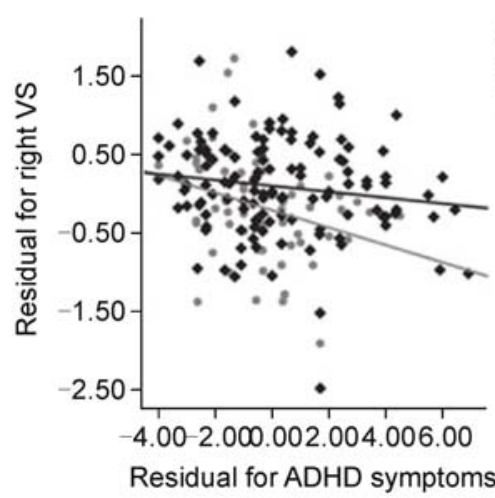

rs12843268

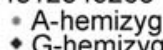

- G-hemizyg
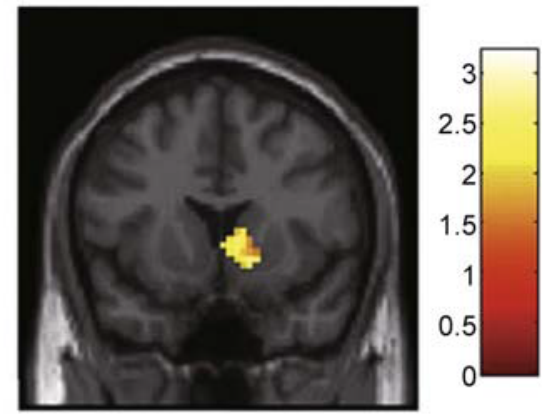

A hemizygotes

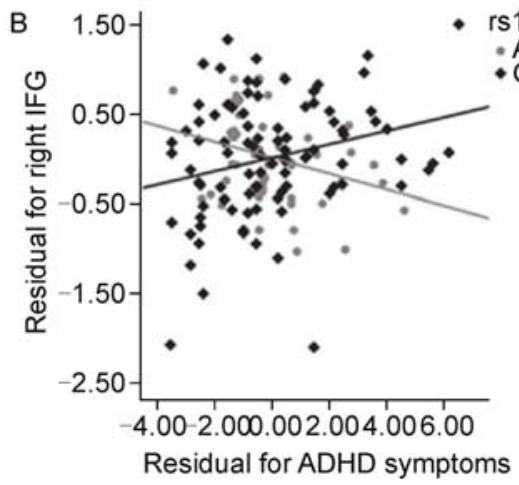

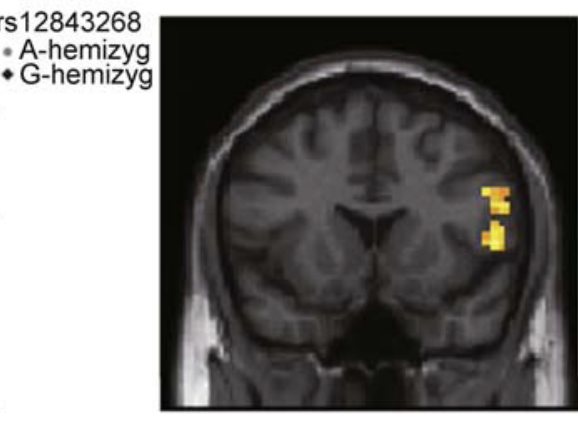

G hemizygotes
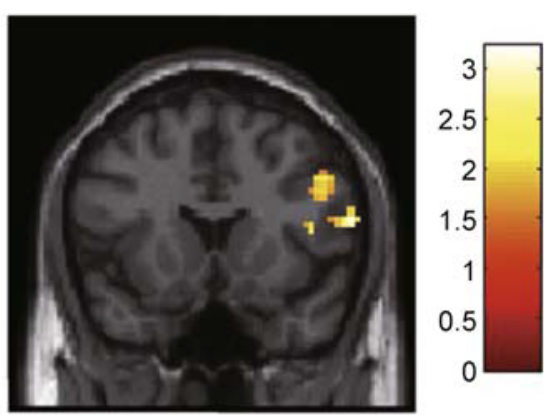

A hemizygotes

Figure 1. Association of ADHD symptoms with distinct BOLD responses depending on MAOA genotype. (A) Significant correlation between right VS activation and ADHD symptoms during reward anticipation in A hemizygotes. (B) Significant positive correlation between right IFG activation and ADHD symptoms during successful stop trials in $\mathrm{G}$ hemizygotes, and significant negative correlation between right IFG activation and ADHD symptoms during successful stop trials in $A$ hemizygotes. Adapted from Nymberg et al. [29] 
Joyner et al. [30] found that common sequence variations in a region in and around gene $M E C P 2$, a well-known determinant of brain structure, show association with cortical surface area in two independent cohorts. Recently, there are also studies that assessed genetic effects on both structural connectivity and functional connectivity [31], and investigated the effect of candidate genes, such as APOE [32] and CNTNAP2 [33], on selected local and global topological measures of anatomical brain networks that characterize the organization of brain wiring.

Gene-environment $(\mathrm{G} \times \mathrm{E})$ interaction is under active investigation as few individual phenotype variations are solely determined by genetics or environment, but a mixture and interaction of both factors. Genetics may not directly influence the development or progression of a brain illness, but modulate the effects of various environmental risk factors on the disease, producing variations across subjects even exposed to the same environment. In general, it is easier to tease apart and estimate genetic and environmental factors along with $\mathrm{G} \times \mathrm{E}$ interaction using a twin or pedigrees design. However, investigating main and interactive genetic and environmental effects in unrelated samples is also possible. In a recent study, Loth et al. [34] investigated the effect of an SNP, located in the oxytocin receptor $(O X T R)$ gene, on the functional MRI BOLD activity in the ventral striatum (VS) and amygdala to animated angry faces, and the association with social-affective problems. They reported that, compared to adolescents with heterozygotic and homozygotic major alleles, adolescents with homozygotic minor alleles had significantly lower VS activity, which was simultaneously associated with more sex-specific emotional problems (girls) and peer problems (boys) in favorable environments, and greater resilience against stressful experiences (Figure 2). These findings partly revealed the $O X T R$-dependent brain mechanisms associated with social-affective problems.

In spite of these advantages and successful examples of candidate-gene candidate-phenotype analysis, a priori knowledge needs to be summarized from reliable results and evidence accumulated in specific areas, and is not always available especially when the underlying mechanisms and the genetic factors of the disease process are largely unknown. One is also faced with the risk to miss potential associations, and is unable to identify novel genetic factors when the search is restricted to a small set of candidate genetic variants and brain regions.

\section{CANDIDATE-GENE WHOLE-BRAIN ANALYSES}

Candidate-gene whole-brain analysis searches the 3dimensional map across many points, surfaces, fiber tracts, or connections in the brain, for potential associations with a handful set of candidate genetic variants. It is a traditional imaging analysis - association tests are performed at each point in the brain independently, producing a statistical parametric map (SPM) [35]. The SPM is then thresholded to identify significant genetic influences. The image of brain features with high spatial resolution makes it possible to precisely localize the genetic effect or map the 3-dimensional profile of the genetic effects in the brain.

A number of studies fall in this category due to the wide availability of standard anatomical MRI images. Filippini et al. [36] investigated the allele-load dependent influences of the best-established genetic risk gene for sporadic Alzheimer's disease (AD), APOE \&4 [37,38], on the regionally specific brain cortical atrophy, and showed distinct patterns of grey matter volume change revealed by different allelic models. Ho et al. [39] studied a variant within the fat mass and obesity-associated gene FTO [40] and localized its effect on structural brain atrophy of the elderly. Mapping genetic effects on brain connectome is also possible [13]. Braskie et al. [41] evaluated the association between $C L U$ risk variant, another strong genetic risk for late-onset $\mathrm{AD}$, and the voxel-wise white matter integrity, measured by FA, in healthy young individuals with DTI and found the most vulnerable brain regions. Westlye et al. [42] tested the hypothesis of $A P O E$-related alterations in resting state functional connectivity. In particular, they performed between-subjects analysis using independent component analysis (ICA) in combination with a dual regression approach [43], which allows for voxel-wise comparisons of resting state functional connectivity. A number of regions were found to have aberrant functional connectivities, which were also correlated with performance on a test of memory functioning. Many other similar studies have mapped the influences of genetic variants in candidate genes, such as $A P O E$ [44], BDNF [45], COMT [46,47], CNTNAP2 [48], MET [49], NTRK1 [50], and ZNF804A [51], on various brain measures.

\section{CANDIDATE-PHENOTYPE GENOME-WIDE ANALYSES}

Genome-wide association studies (GWAS) had been widely performed on disease status, or diagnostic, cognitive and behavioral measures before imaging traits were introduced as endophenotypes and have been discovering new genetic loci which might influence the risk of a certain disease in recent years $[52,53]$. Candidate-phenotype genome-wide analysis is a traditional genome-wide association study (GWAS). When a single measure derived from brain images is known to be an outstanding biomarker for a particular mental disorder 

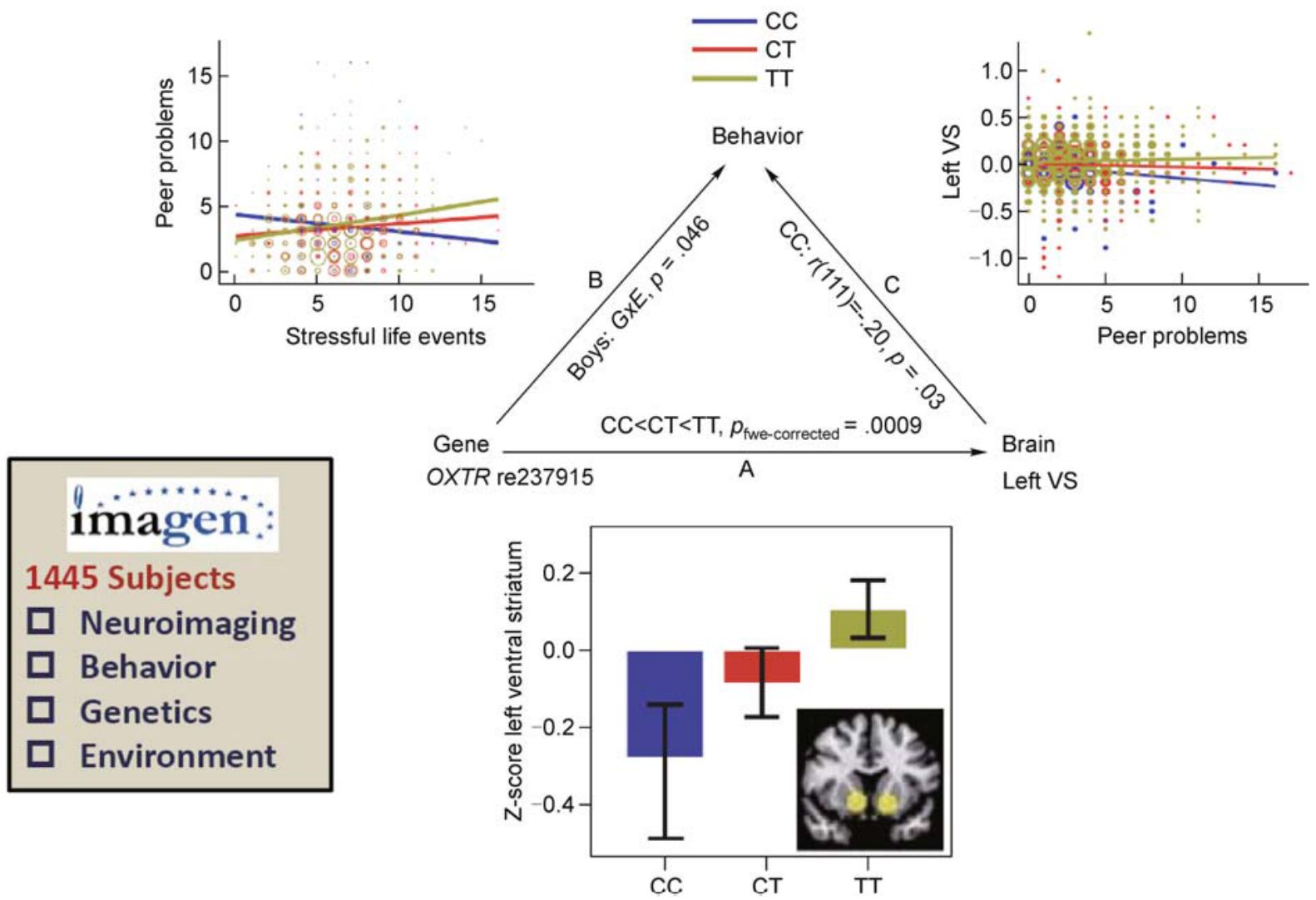

Figure 2. Path diagram of the associations between $O X T R$ rs237915, left ventral striatum (VS) activity and peer problems in boys. (A) The effect of rs237915 on left VS activity. Minor allele homozygotes (CC) have reduced VS activity. (B) rs237915genotype $\times$ stressful life events (SLE) interaction effect on peer problems in boys. Male CC-carriers show greater resilience against stressful experiences but have more peer problems than CT/TT carriers in low-SLE environments. (C) CC-carriers show a significant correlation between lower left VS activity and increased peer problems. Adapted from Loth et al. [34]

or biological process, a whole genome screening identifies genetic variants that are most associated with the imaging trait. A $p$-value less than the nominal $5.0 \times$ $10^{-8}$ is commonly used to reject the null hypothesis of no genetic effect. Follow-up analyses can then be performed to identify the causal genes, and investigate a set of biological processes that the causal genes are involved, such as the protein expressed by the genes, or the interaction of the genes.

There are also quite a few studies in this category as genome-wide data has become far more cost effective to acquire. For example, Potkin et al. [54] discovered the association of two genes with the BOLD contrast activation in the left dorsal lateral prefrontal cortex measured during a working memory task in schizophrenia patients, and verified the finding in an independent study. Stein et al. [55] identified genetic variants associated with the highly heritable caudate volume and cross-validated the results in two large cohorts. Other GWAS using quantitative brain imaging phenotypes measuring the structure, function, and anatomical connectivity in the brain have also been conducted [56-59]. The imaging phenotype does not need to be restricted to a single brain measure. Shen et al. [60] split the brain into 142 cortical and subcortical regions, and extracted summary measures of grey matter density, volume, and cortical thickness from each of the regions using voxel-based morphometry (VBM) [5]. These region-specific imaging traits were then fed into genome-wide association scans. Several SNPs and genes were shown to be associated with the vulnerability to structural change in multiple brain regions. Parcellating the brain into a number of regions based on an atlas and deriving summary measures for each region make it feasible to perform a coarse wholebrain genome-wide association study. Similarly, Jahanshad et al. [61] divided the cortex into areas of known structure and function, and mapped white matter fiber pathways between pairs of brain regions with high- 
resolution tractography. GWAS was then performed and replicated on 59 anatomical connectivities with moderate to high heritability. Secondary GWAS analyses on several organizational and topological network measures characterizing the weighted brain anatomical networks discovered variants significantly associated with local properties of the network. This study can be viewed as a coarse connectome-wide genome-wide association study. To date, few studies search the entire connectome and genome for association. This will be a promising direction in the future.

\section{WHOLE-BRAIN GENOME-WIDE ANALYSES}

Unlike traditional association studies that test the association between a single trait and many genetic variants, brain imaging data provides a trait at each voxel in the brain, each vertex on a surface, or each connection in a brain network. The trend in imaging genetics may be to embrace the whole-brain, genome-wide association paradigm, where both the entire brain and entire genome are searched for non-random associations. This brings unprecedented opportunities to identify novel genetic variants underpinning imaging measures and map their 3dimensional spatial effects in the brain. However, the dramatically increased dimensionality of the problem also imposes many challenges on the attempt to do wholebrain genome-wide searches, and makes most conventional methods have a low statistical power. We now review the rapidly evolving methodological approaches in this category.

\section{Univariate-imaging univariate-genetic association}

Stein et al. [62] were the first to perform a completely unbiased whole-brain genome-wide association study. They explored the relation between 448293 SNPs and 31622 voxels in whole-brain maps of local brain volumetric change calculated by tensor-based morphometry (TBM) [63] across 740 elderly subjects from the Alzheimer's Disease Neuroimaging Initiative (ADNI) (http://adni.loni.ucla.edu). Massive univariate association tests were performed for each pair of the SNPs and voxels, resulting in a total of approximately $1.4 \times 10^{10}$ statistical tests. The heavy computational burden was handled by paralleling the computation across 300 cluster nodes. To accommodate the huge number of statistical tests performed, only the $p$-value for the most associated SNP across the genome was recorded at each voxel, and thus the null distribution for the $p$-value at each voxel follows a Beta distribution, which was used to theoretically adjust the $p$-values. Several top genetic variants were identified with biological interest, suggesting further explorations, but no SNP had whole-brain whole-genome significance.

This work is pioneering and is a proof of concept as it shows that a completely unbiased scan of the whole brain and the entire genome is feasible. However, the drawbacks of this approach are also clear. Univariate-imaging univariate-genetic association tests completely ignore the spatial correlation in 3-dimensional imaging data and the LD structure along the genome. Since voxel-wise brain measures are noisy, and a single common variant normally has only very small effect on brain structure, massive univariate approaches, which fail to borrow information from neighboring voxels or nearby genetic variants, typically have poor reproducibility and low power. Also, with millions of billions of statistical tests to perform, the computational burden is extremely heavy, and the colossal multiple comparisons correction often leaves no significant associations [62]. Clearly, more sophisticated multivariate methods [64] are needed to account for the correlation structures in both imaging and genetic data.

\section{Univariate-imaging multivariate-genetic association}

Multivariate methods can be used to combine the effects from multiple SNPs and model their joint effect on imaging traits. Multivariate genetic approaches typically use a priori information on the gene or haplotype structure to group SNPs into a multivariate statistical model. SNP sets can be formed by SNPs located in or near a gene, SNPs located within a gene pathway, SNPs within evolutionary conserved regions, or other a priori biological information. Alternatively, the grouping may be based on a sliding window with an appropriate window size or the haplotype blocks to provide a full coverage of the entire genome [65]. Performing set-based association tests combines nearby SNPs to model their joint effect, reduces the total number of statistical tests, and thus alleviates the stringent multiple testing correction compared to individual-SNP tests. Overall, multivariate methods often have improved reproducibility and increased power relative to univariate methods, especially when SNPs with similar but modest effects that are hard to detect individually are grouped and modeled jointly.

Early work on set-based tests combines test statistics or $p$-values from standard individual-SNP tests by making use of the gene annotation information and the estimated LD structure $[66,67]$. These methods heavily rely on individual-SNP tests and therefore suffer from many of the same problems as univariate tests. For example, they cannot accommodate interactions between SNPs and have low power when SNPs are not in LD with the causal variants. 
A natural and classical way to model the overall effect of multiple SNPs is to construct a multiple regression model. However, high LD between co-segregated SNPs in haplotype blocks [23] often leads to collinearity between the SNP regressors and can produce unstable parameter estimates and substantially overestimated degrees of freedom. If it is reasonable to assume that all the genetic variants in a chromosomal region are causative and affect the phenotype in the same direction with similar magnitudes, a burden test can be performed which collapses or summarizes the SNP information in a genetic region into a single burden regressor. For example, the burden regressor could be a single dichotomous variable for each subject, indicating whether or not the subject has any rare variants within a region (cohort allelic sum test, CAST [68]), or a single weighted average (based on MAF) of the number of minor alleles for each individual (weighted sum test, WST [69]), or a single regressor indicating the number of rare variants in a genetic region for each individual [70]. It is also possible to form several burden regressors and then perform a standard multiple regression [71]. Alternatively, high dimensional variable selection techniques could be used to handle the collinearity of the SNP regressors. These include the penalized and sparse regression techniques such as the ridge regression [72,73], the least absolute shrinkage and selection operator (LASSO) [74,75], and elastic net $[76,77]$. These regularized regression methods allow for a large number of correlated SNPs being incorporated in a single model and have detection power by selecting a sparse set of SNPs that are associated with imaging measures.

Hibar et al. [78] applied the principal components regression (PCReg) to approach the collinearity problem. PCReg is a method that first performs principle component analysis (PCA) on the set of SNP regressors to extract mutually orthogonal predictors that explain a large majority of the overall variance as the original regressors, and then applies a standard partial $F$-test with the orthogonal regressors, and thereby balances the total variance explained and the degrees of freedom of the model. With this technique, Hibar et al. [78] was able to carry out a voxel-wise gene-wide association study (vGeneWAS) using the same imaging and genetic data as in Stein et al. [62] by grouping SNPs into 18044 genes based on gene membership. They showed the increased power of their methods compared to vGWAS [62] due to the multivariate modeling of the SNPs and a substantially reduced number of statistical tests. However, no gene was identified with whole-brain genome-wide significance, perhaps due to the over-simplification of the empirical and linear method, and the massive univariate nature of the method on brain images.

Ge et al. [79] introduced to imaging genetics literature a semi-parametric kernel machine based multi-locus model $[80,81]$, which provides a biologically-informed way to capture the interactions between SNPs and model their joint effect on imaging traits. This method is easy to adjust for non-SNP covariates and offers a flexible framework to model epistatic effects between genetic variants based on the choice of kernels, whose elements are measures of genetic similarity between pairs of subjects. By using nonparametric kernels, the method can in principle capture any types of SNP-SNP interactions [82], and is more powerful relative to burden test when a genetic region has both protective and deleterious variants or many non-causal variants. Recently, there is an increasing recognition that rare variants (with MAFs $<1 \%-5 \%$ ) are important for complex disease, and it appears that most imaging traits are influenced by many common variants with relatively small effects and a handful of rare variants with larger effects. An attracting feature of the kernel method is that it is capable of testing cumulative effects of rare variants in genetic regions or SNP sets. By introducing appropriate weights in the kernel function when combining SNPs, one can even up-weight rare variants and down-weight common variants using a priori knowledge as similarity in rare alleles might be more informative than similarity in common alleles. Lastly, by using a connection to linear mixed models, the semiparametric multi-locus model can be fitted efficiently at each voxel, and a standard variance component test [83], termed as a sequence kernel association test (SKAT), can be used to make inference, yielding an approximate chisquared statistical map whose degrees of freedom can adapt to the correlation structure of the SNP sets [80]. In combining this kernel method with a suite of other approaches, a head-to-head comparison with vGWAS [62] and vGeneWAS [78] using exactly the same data set showed boosted statistical power. Several genes were identified with whole-brain whole-genome significance for the first time (Figure 3) [79].

\section{Joint multivariate association}

To account for the multivariate nature and exploit the covariance structure of both imaging and genetic data, joint modeling of the imaging and genetic data appears to be promising. One possible candidate in this category is a regularized version of the two-block method, e.g., the canonical correlation analysis (CCA) [84] and the partial least squares (PLS) regression [85] with an additional $1^{1}$ or $1^{2}$ regularization to handle high dimensional data and perform variable selection. Both methods hypothesize that imaging and genetic data are linked through two unobserved latent variables, and seek linear combinations of the two data blocks - as an approximation to the latent variable - that possess the maximum correlation 


\section{CRIN2B}
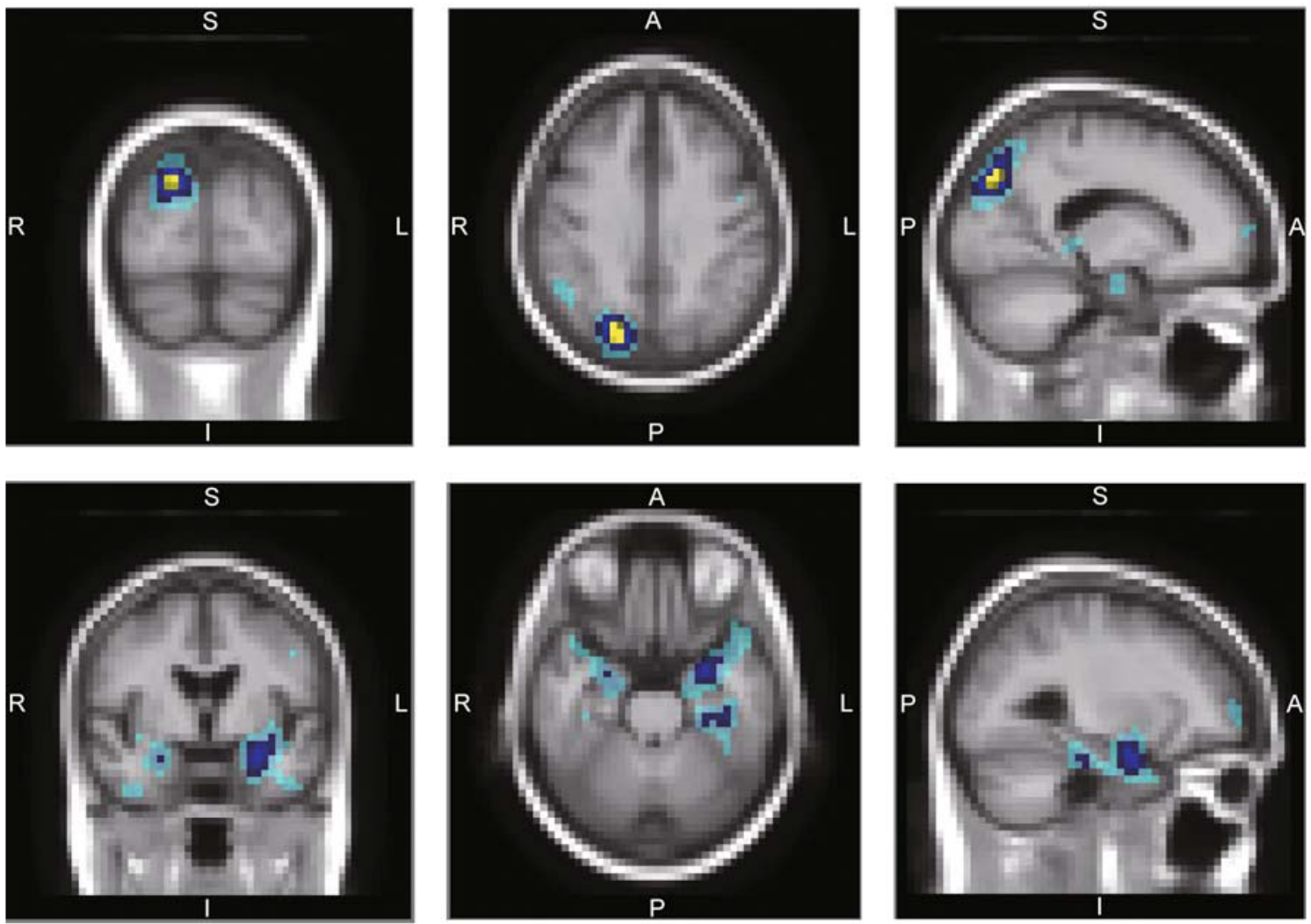

Figure 3. Results from the whole-brain genome-wide association study using the kernel machine based multi-locus model. The parietal (upper panels) and temporal (lower panels) foci are influenced by the most associated gene GRIN2B. Brainwide genome-wide significant voxels are in yellow; brain-wide (post hoc gene-wise) significant voxels are in dark blue; and 0.001 uncorrected significant voxels are in light blue. Both parietal and temporal lobes are known to be affected in Alzheimer's disease. Adapted from Ge et al. [79]

(CCA) or covariance (PLS) with each other. Recently, sparse CCA and sparse PLS have been applied to imaging genetics respectively $[86,87]$.

Liu et al. [88] proposed another method to link the two blocks of data known as parallel independent component analysis (paraICA or PICA). The method discovers independent components of the imaging and genetic data respectively, and at the same time selects the components of the two modalities by maximizing a correlation measure. One challenge of this approach is that it may be hard to recover the contributing SNPs and localize the spatial effect in the brain from large genetic and imaging components, making the results hard to interpret. In addition, the power of the method to detect associations may be reduced when there is substantial amount of noise from the genetic data.

An alternative perspective on handling the two blocks of data is to consider a multivariate multiple regression, $i$. e., regressing the entire imaging data block on the genetic data, and impose different structures or regularizations on the regression coefficient matrix. Recent works in this category include group-sparse multi-task regression [89] and sparse multi-modal multi-task regression [90], which incorporate the grouping of SNPs induced from their genetic arrangement and enforce sparsity across different SNP groups. The combined structured sparsity regularizations also allow for identifying multi-modal biomarkers and their shared underlying genetic factors. Also within the multivariate multiple regression framework, Vounou et al. [91] proposed a sparse reduced-rank regression (sRRR) method. sRRR reduces the rank of the regression coefficient matrix to a number much smaller than the number of imaging traits and the number of SNPs, and then factorizes the coefficient matrix into the product of two small full-rank matrices, which are imposed with structures for gene/pathway grouping and constrained to be sparse. Vounou et al. [91] showed the boosted sensitivity of sRRR in simulation studies with an increased number of subjects and SNPs relative to massive univariate methods. The method was applied to 
real whole-brain whole-genome data sets in subsequent publications $[92,93]$.

Joint multivariate methods respect and exploit the multivariate nature of the imaging and genetic data, and substantially reduce the number of statistical tests, which alleviates the multiple testing correction problem. Therefore, they may provide increased statistical power relative to univariate-imaging or univariate-genetic methods. However, joint modeling of very high dimensional data also brings both computational and statistical challenges. Sparse regularization methods normally employ iterative optimization procedures, so the computational demand is high, especially when one needs to tune some regularization parameters and to validate the results through crossvalidation or permutation schemes. Over-fitting is also a common issue in handling high dimensional data, which may result in a reduced power of the methods. Currently, in most applications, considerable downsampling of the imaging and genetic data is needed before they are fed into these multivariate models.

In addition to machine learning approaches, recently, there are efforts to develop more comprehensive probabilistic models for the joint modeling of imaging and genetic data. Stingo et al. [94] presented a Bayesian hierarchical modeling approach for imaging genetics, which incorporates the selection of brain regions that discriminate patients and healthy controls. Genetic data were linked to the observed imaging features by specifying group-specific distributions that are dependent on model selected SNPs. They also imposed a neighborhood structure on the brain regions based on their spatial locations, and applied the random Markov field theory to incorporate prior knowledge on the connectivity among brain regions. When applied to real imaging genetic data, the model allows for simultaneous selection of a set of discriminatory brain regions and the relevant SNPs, together with the reconstruction of the dependence structure of the selected regions. Batmanghelich et al. [95] proposed a unified Bayesian framework for the joint modeling of genetic variants, imaging traits, and the disease phenotype. The generative model captures the overall process via a logistic regression, which predicts disease status from imaging traits, and a ridge regression, which associates genetic variants to imaging features. With appropriately specified priors, the model is able to select a small set of genetic variants that induce the variation in certain representative imaging traits, which in turn exhibit discriminative capability with respect to the disease phenotype. Thus the model establishes the pathway among genetics, imaging features and disease status with associated probability measures.

Joint modeling of imaging and genetic data, and even disease phenotypes using rigorous modeling strategies is a promising direction as it accounts for, to some extent, the underlying mechanisms of the observations, incorporates prior information, and better captures intrinsic structures of imaging and genetic data, and thus provides a more comprehensive way to integrate multi-modal data than many machine learning approaches. The only issue is that these methods typically involve sophisticated inference methods, such as Markov chain Monte Carlo (MCMC), which require intensive computation, and therefore need a balance between model flexibility and computational burden.

\section{DATA REDUCTION AND MULTIPLE TESTING CORRECTION}

\section{Data reduction methods}

The ultra-high dimensional imaging and genetic data imposes many challenges on whole-brain genome-wide modeling, and often causes statistical and computational problems. Therefore, a balance is often needed between pure discovery methods and those that invoke data reduction. A priori hypotheses or biological information may be used to constrain the analysis to a handful of brain regions or a wide list of potentially associated SNPs and genes. Alternatively, various software packages and atlases may be used to parcellate the brain into a number of cortical and subcortical regions of interest (ROI), and derive a summary measure from each ROI to get a coarse coverage of the entire brain. Such parcellation is easy to perform and consistent across subjects, but has the risk of missing patterns of effects that lie only partially within the chosen ROIs. Data-driven feature extraction methods such as principal component analysis (PCA) and independent component analysis (ICA) may be used to avoid these problems, but may raise difficulties to align extracted features across subjects. Recently, Chiang et al. [58] proposed a novel approach to reduce the dimensionality of voxel-wise data. Specifically, they selected highly genetically influenced voxels and then grouped these voxels into ROIs using hierarchical clustering based on their genetic correlation within images. This approach has the advantage of defining ROIs whose component voxels are influenced by a highly overlapping set of genes, and can potentially be applied to any imaging modalities that show pleiotropy. As for the data-driven dimension reduction of the whole-genome data, a preliminary univariate filtering is usually applied. Multivariate methods may also be used iteratively, removing the lowest ranked variables at each iteration [96]. Iterative sure independence screening $[97,98]$ iterates a univariate screening procedure conditional on the previously selected features to capture important features that are 
Table 2. Advantages and disadvantages of various imaging genetic association methods

\begin{tabular}{|c|c|c|c|}
\hline \multicolumn{2}{|c|}{ Imaging genetic association methods } & \multirow{2}{*}{$\begin{array}{l}\text { Advantages } \\
\text { Test biologically plausible } \\
\text { hypotheses; clearly interpretable } \\
\text { results; well established statistical } \\
\text { methods }\end{array}$} & \multirow{2}{*}{$\begin{array}{l}\text { Disadvantages } \\
\text { Need strong a priori knowledge; low } \\
\text { potential for novel findings }\end{array}$} \\
\hline $\begin{array}{l}\text { Candidate } \\
\text { approaches }\end{array}$ & $\begin{array}{l}\text { Candidate-gene candidate-phenotype } \\
\text { analyses/Candidate-gene whole-brain } \\
\text { analyses/Candidate-phenotype } \\
\text { genome-wide analyses }\end{array}$ & & \\
\hline \multirow{5}{*}{$\begin{array}{l}\text { Whole-brain } \\
\text { genome-wide } \\
\text { analyses }\end{array}$} & vGWAS & $\begin{array}{l}\text { Completely unbiased scan of } \\
\text { voxel-wise genome-wide data }\end{array}$ & $\begin{array}{l}\text { Ignore spatial correlation and LD struc- } \\
\text { ture; poor reproducibility, low power; } \\
\text { heavy computational burden \& multiple } \\
\text { testing correction }\end{array}$ \\
\hline & $\begin{array}{l}\text { Regularized multiple regression/ } \\
\text { PCReg }\end{array}$ & $\begin{array}{l}\text { Model the overall effect of multiple } \\
\text { SNPs; reduce multiple comparison } \\
\text { problem }\end{array}$ & $\begin{array}{l}\text { Ignore spatial correlation; unable to } \\
\text { capture complex SNP-SNP interactions }\end{array}$ \\
\hline & Kernel methods and RFT & $\begin{array}{l}\text { Make use of spatial information; } \\
\text { capture any types of SNP-SNP } \\
\text { interactions; test effects of rare } \\
\text { variants; computationally efficient }\end{array}$ & $\begin{array}{l}\text { Assumptions needed for parametric } \\
\text { methods being valid and accurate }\end{array}$ \\
\hline & $\begin{array}{l}\text { Regularized two-block methods/ } \\
\text { regularized multivariate multiple } \\
\text { regression / paraICA }\end{array}$ & $\begin{array}{l}\text { Respect multivariate nature of } \\
\text { imaging and genetic data; } \\
\text { dramatically reduce multiple } \\
\text { comparison problem }\end{array}$ & $\begin{array}{l}\text { High computational demand; over-fitting } \\
\text { issues; results may be hard to interpret }\end{array}$ \\
\hline & Bayesian probabilistic modeling & $\begin{array}{l}\text { A comprehensive way to integrate } \\
\text { multi-modal data; offer model } \\
\text { flexibility and interpretability power; } \\
\text { incorporation of prior information }\end{array}$ & $\begin{array}{l}\text { Typically involve sophisticated infer- } \\
\text { ence methods }\end{array}$ \\
\hline
\end{tabular}

marginally uncorrelated with response. This may be a promising method for genetic data reduction and has been applied to genome-wide association studies [99].

\section{Multiple testing correction}

Seeking potential associations between imaging traits and genetic variants using univariate-imaging or univariategenetic analyses typically involves performing a large number of statistical tests at each voxel in the brain or for each genetic variant on the genome. In both contexts, multiple testing correction must be made to account for the number of statistical tests and control false positives.

When multiple testing correction is needed over the brain, i.e., to threshold the SPM and localize significant genetic influences, traditional methods such as Bonferroni correction and false discovery rate (FDR) procedures $[100,101]$ may be used. However, while they are sensible ways to control false positives, these voxel-wise correction methods do not account for the spatial correlation structure in imaging data and thus often lack power. If one needs only to map the 3-dimensional genetic influences for a small number of genetic variants, nonparametric permutation methods $[102,103]$ may be an optimal choice, as it requires few assumptions and allows for the consideration of nonstandard test statistics when no parametric result is available. However, the heavy computational burden of permutation tests prevents its application to more than a handful of genetic variants.
Sometimes, under appropriate assumptions, pooling and parametric fitting [104] of permutation distributions offer the potential to make permutation practical [79]. Random field theory (RFT) is a core inferential tool in neuroimaging [35]. It assesses the behavior of extreme test statistic value or spatial extent of a set of contiguous voxels exceeding some predefined threshold in an elegant way [105], and has a unified theory for various statistical maps $[106,107]$. Ge et al. [79] were the first to overcome the time consuming smoothness estimation process, and applied a fast implementation of RFT to a voxel-wise genome-wide data set. RFT makes use of the spatial information in imaging data by implicitly accounting for the search volume and the smoothness of the statistic images, and produces more sensitive family-wise error (FWE) corrected inferences than voxel-wise correction methods. Cluster-inspired methods other than standard peak and cluster size inferences such as threshold-free cluster enhancement [108] and RFT cluster-mass test [109] may also be useful. A variant that combines RFT and FDR, known as topological FDR, has also been developed [110]. With a similar spirit as RFT to make use of spatial information, Zhang et al. [111] proposed a novel FDR procedure that embeds the information of neighboring voxels into the conventional FDR to improve sensitivity and enhance signal detection.

Multiple testing correction over the genome is also not straightforward as the correlation structure along the genome is complex and unpredictable. In screening the 
genome for association, the number of SNPs often substantially outnumbers the independent statistical tests actually performed, making the Bonferroni correction or standard FDR procedure over-conservative. One widely used approach is to apply a PCA on the LD matrix between SNPs and set the effective number of independent tests as the number of principal components required to jointly explain a large majority (e.g., 99.5\%) of the overall variance in the original SNP sets $[112,113]$. A standard Bonferroni or FDR procedure can then be applied, replacing the total number of SNPs by the effective number of independent tests. Recently, following a similar idea but with full statistical rigor, Fan et al. [114] proposed a novel method based on principal factor approximation that can accurately estimate false discovery proportion under an arbitrary dependence structure. Multi-dimensional local FDR procedures [115] may also be promising to capture different aspects of the information contained in genetic data.

Finally, with the increasing popularization of graphic processing unit (GPU) that provides orders of magnitude speed-ups [116], large-scale resampling-based tests will become practically feasible. However, Bayesian probabilistic modeling, as a completely different direction, which assigns probabilistic measures of relevance to each pair of genetic variants and imaging traits, and is free of multiple testing correction problems, also has its place as it can provide more information, e.g., the spatial confidence intervals, on genetic effects of interest, incorporate prior information, offer more flexible and interpretable model estimates, and facilitate model comparison and selection [117].

\section{CONCLUSION AND FUTURE DIRECTIONS}

Although imaging genetic studies have been discovering novel genetic loci and imaging markers that are associated with brain illnesses, it has been increasingly recognized that replications are particularly important in discovery science such as imaging genetics to lower false positives and spurious findings [118]. However, unlike many studies in psychiatric genetics that involve tens of thousands of replication samples to facilitate replication [119], a majority of the imaging genetic association studies are still limited to hundreds rather than thousands of subjects. Moreover, genetic variants typically have only subtle effects on imaging measures (influencing the phenotypic variance less than $1 \%$ per allele), much lower than the effect of brain activity evoked by external stimuli, or morphometric change induced by some disease processes [13]. Therefore, replications in imaging genetic studies are faced with more difficulties than in imagingonly or genetic-only studies. To address the nonreplication issue, a few large imaging genetics data sets have been collected in the past few years and made publicly available. For example, since 2005, the longitudinal Alzheimer's Disease Neuroimaging Initiative (ADNI; http://adni.loni.ucla.edu/) has been collecting and releasing MRI and PET images, whole-genome genetics, cognitive tests, and other biomarkers of Alzheimer's Disease (AD) from hundreds of AD patients, mild cognitive impairment (MCI) subjects and elderly controls with an aim to define the progression of $\mathrm{AD}$ and validate the use of various biomarkers for $\mathrm{AD}$ diagnosis and treatment. The IMAGEN study (http: //www.imageneurope.com/) is another large-scale multi-center imaging genetic consortium aiming at identifying the genetic and neurobiological basis of individual variability in impulsivity, reinforcer sensitivity and emotional reactivity, and determining their predictive capability for the development of frequent psychiatric disorders [120]. Multi-modal data including MRI, functional MRI, genome-wide genetics were collected from 2000 14-year-old adolescents with longitudinal follow-ups. A more recent endeavor along this line is an EU multi-million collaborative project IMAGEMEND (IMAging GEnetics for MENtal Disorders). IMAGEMEND focuses on schizophrenia, bipolar disorder and attention deficit/ hyperactivity disorder (ADHD), and aims to assemble Europe's largest data set, combining imaging, genetic, environmental, cognitive and clinical information. This consortium, comprising data from multiple disorders, allows for the development of trans-diagnostic models in order to uncover shared genetic and environmental risk factors, and fundamental processes underlying multiple mental disorders, and helps understand comorbidity among disorders. These freely available large data sets, collected and preprocessed following standard protocols and procedures, will undoubtedly keep processing errors to the minimum and improve reliability and credibility of the findings.

Meta-analytic studies, which synthesize and combine imaging genetic findings from many cohorts worldwide to increase statistical power and identify consistent genetic effects across studies, can to some extent resolve the replication problem and have attracted increasing interest. The ENIGMA Consortium (Enhancing Neuro Imaging Genetics Through Meta-Analysis; http://enigma.loni. ucla.edu) pools data from imaging genetic studies to offer the power to detect robust common genetic associations. As a successful example, Stein et al. [121] pooled data on hippocampal volume and intracranial volume from 21151 individuals scanned at 125 institutions worldwide, and identified several common genetic variants that influence these brain structures. Follow-up efforts screening the genome for common genetic influences on subcortical structures as well as highresolution FA images [122] are underway. Interactive 
tools to visualize the ENIGMA association results (EnigmaVis) are also under active development [123].

To summarize, imaging genetics is still in its infancy but promises to shed light on how genetic variants influence brain organization and function. Statistical approaches are rapidly evolving from univariate analyses to multivariate analyses, and from candidate approaches towards complete discovery science. Computationally efficient, biologically plausible, and statistically powerful methods are needed to handle the very high dimensional imaging and genetic data with complex covariance and noise structures. International joint efforts and collaborations are needed to improve the reliability, incredibility and reproducibility of the findings through data sharing, phenotypic harmonization procedures and meta-analysis. The desire to understand the human brain will never come to an end. Very recently, the European Union (EU) and the US have successively launched the Human Brain Project (HBP) and the BRAIN Initiative (Brain Research through Advancing Innovative Neurotechnologies) respectively. Both projects have the ambition to simulate and mimic the human brain with supercomputers, and even map the activity of every single neuron, in order to gain profound insight into how the human brain is functioning, and to better diagnose brain illnesses and develop new treat- ments. With the ever largest investment in brain science from EU and the US government - approximately one billion euro in funding for HBP and three billion dollars for the BRAIN over ten years, the gap between brain activity at the level of single neurons and the measurements at the level of brain regions using functional brain imaging techniques will be largely filled.

Eventually, the 5-O (GenO-, NeurO-, EndophenO-, BehaviO-, and EnvirO-) approach will open a new era to the investigation of mental disorders. As shown in Figure 4, big data will first be collected and analyzed at the genotypic (e.g., SNPs, CNVs, etc.), neuronal (e.g., electrophysiological recordings, animal models, etc.), endophenotypic (e.g., features extracted from structural, functional, and diffusion images), behavioral (e.g., various neuropsychiatric rating scales), and environmental (e.g., negative experience, stress, etc.) level. More importantly, the 5-O approach refers to integration of the data and results across multiple levels and modalities using multi-level multi-modal modeling and cutting-edge computational tools, to identify biomarkers for various neuropsychiatric disorders, reveal their genetic basis, account for their underlying biological processes and mechanisms, as well as their interaction with environmental factors. Well-developed animal models will help

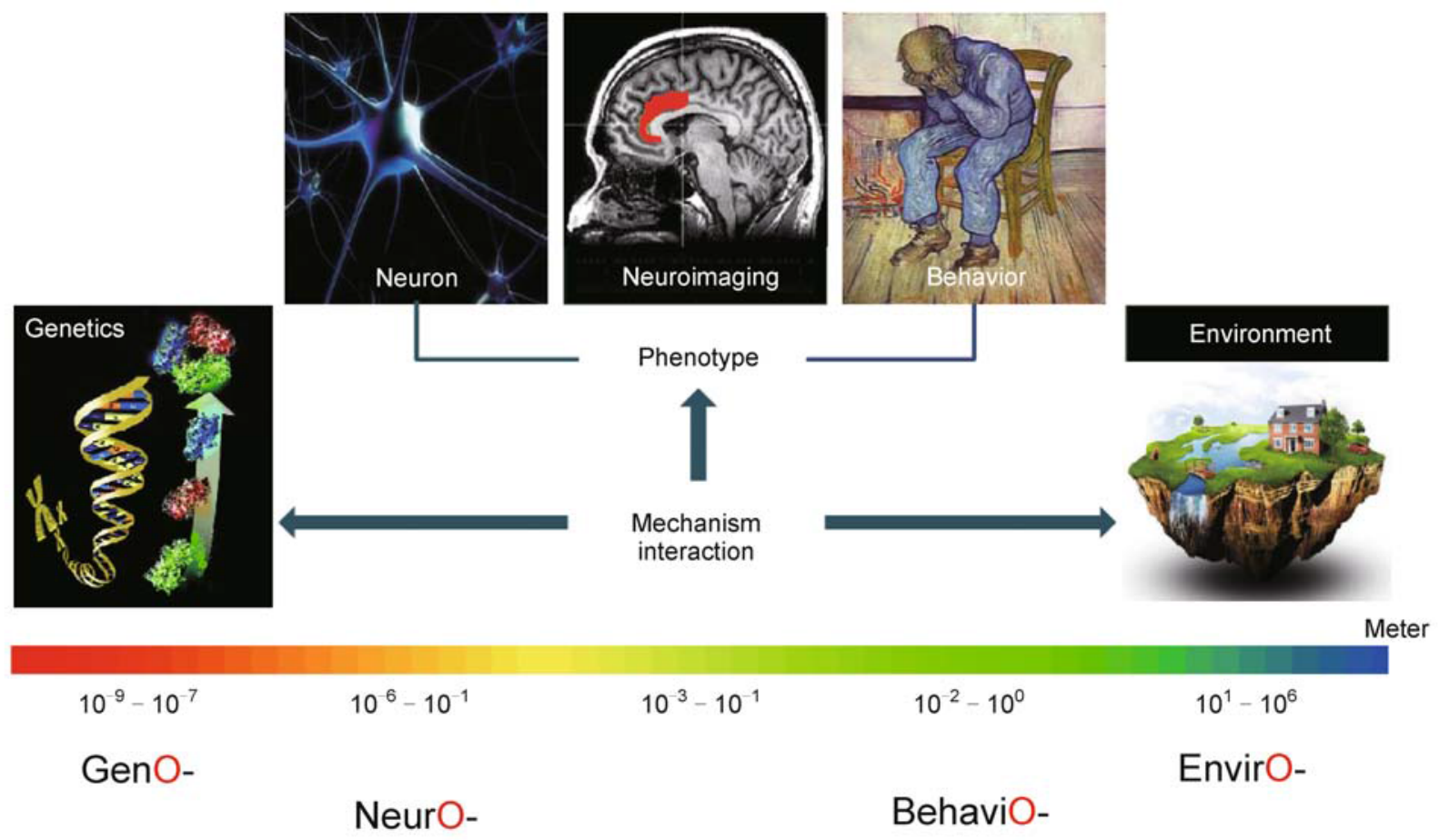

EndophenO-

Figure 4. The 5-O (GenO-, NeurO-, EndophenO-, BehaviO-, and EnvirO-) approach for the investigation of mental disorder. Multi-level multi-modal data are collected and integrated by comprehensive modeling and cutting-edge computational tools, to identify biomarkers for various neuropsychiatric disorders, reveal their genetic basis, account for their underlying biological processes and mechanisms, as well as their interaction with environmental factors. 
cross-validate the findings. The gap between genetics, brain images, and behaviors will be largely bridged, providing deep insight into how genetic variants underpin various neuropsychiatric disorders, and depicting a complete picture of the pathways through which genetic factors exert their effects on the brain. We would expect that we will be getting increasingly close to improve automated diagnosis at the pre-symptomatic stage, better monitor and predict disease progression and treatment response, develop personalized treatment, and finally reach full clinical practice in psychiatry.

\section{CONFLICT OF INTERESTS}

The author Tian Ge, Gunter Schumann and Jianfeng Feng declare that they have no conflict of interests.

\section{REFERENCES}

1. Gottesman, I. I. and Shields, J. (1972) Schizophrenia Genetics: A Twin Study Vantage Point. New York: Academic Press

2. Gottesman, I. I. and Gould, T. D. (2003) The endophenotype concept in psychiatry: etymology and strategic intentions. Am. J. Psychiatry, $160,636-645$

3. Meyer-Lindenberg, A. and Weinberger, D. R. (2006) Intermediate phenotypes and genetic mechanisms of psychiatric disorders. Nat. Rev. Neurosci., 7, 818-827

4. Akil, H., Brenner, S., Kandel, E., Kendler, K. S., King, M. C., Scolnick, E., Watson, J. D. and Zoghbi, H. Y. (2010) Medicine. the future of psychiatric research: genomes and neural circuits. Science, $327,1580-1581$

5. Ashburner, J. and Friston, K. J. (2000) Voxel-based morphometry - the methods. Neuroimage, 11, 805-821

6. Dale, A. M., Fischl, B. and Sereno, M. I. (1999) Cortical surface-based analysis. I. Segmentation and surface reconstruction. Neuroimage, 9, 179-194

7. Fischl, B., Sereno, M. I. and Dale, A. M. (1999) Cortical surface-based analysis. II: Inflation, flattening, and a surface-based coordinate system. Neuroimage, 9, 195-207

8. Fischl, B. (2012) FreeSurfer. Neuroimage, 62, 774-781

9. Rimol, L. M., Panizzon, M. S., Fennema-Notestine, C., Eyler, L. T., Fischl, B., Franz, C. E., Hagler, D. J., Lyons, M. J., Neale, M. C., Pacheco, J., et al. (2010) Cortical thickness is influenced by regionally specific genetic factors. Biol. Psychiatry, 67, 493-499

10. Winkler, A. M., Kochunov, P., Blangero, J., Almasy, L., Zilles, K., Fox, P. T., Duggirala, R. and Glahn, D. C. (2010) Cortical thickness or grey matter volume? The importance of selecting the phenotype for imaging genetics studies. Neuroimage, 53, 1135-1146

11. Fox, M. D. and Raichle, M. E. (2007) Spontaneous fluctuations in brain activity observed with functional magnetic resonance imaging. Nat. Rev. Neurosci., 8, 700-711

12. Smith, S. M., Fox, P. T., Miller, K. L., Glahn, D. C., Fox, P. M., Mackay, C. E., Filippini, N., Watkins, K. E., Toro, R., Laird, A. R., et al. (2009) Correspondence of the brain's functional architecture during activation and rest. Proc. Natl. Acad. Sci. U.S.A., 106, 13040-13045

13. Thompson, P. M., Ge, T., Glahn, D. C., Jahanshad, N. and Nichols, T. E. (2013) Genetics of the connectome. Neuroimage, $80,475-488$
14. Chiang, M. C., Barysheva, M., Shattuck, D. W., Lee, A. D., Madsen, S. K., Avedissian, C., Klunder, A. D., Toga, A. W., McMahon, K. L., de Zubicaray, G. I., et al. (2009) Genetics of brain fiber architecture and intellectual performance. J. Neurosci., 29, 2212-2224

15. Kochunov, P., Glahn, D. C., Lancaster, J. L., Winkler, A. M., Smith, S., Thompson, P. M., Almasy, L., Duggirala, R., Fox, P. T. and Blangero, J. (2010) Genetics of microstructure of cerebral white matter using diffusion tensor imaging. Neuroimage, 53, 1109-1116

16. Thomason, M. E. and Thompson, P. M. (2011) Diffusion imaging, white matter, and psychopathology. Annu Rev Clin Psychol, 7, 63-85

17. Jbabdi, S. and Johansen-Berg, H. (2011) Tractography: where do we go from here? Brain Connect, 1, 169-183

18. Glahn, D. C., Winkler, A. M., Kochunov, P., Almasy, L., Duggirala, R., Carless, M. A., Curran, J. C., Olvera, R. L., Laird, A. R., Smith, S. M., et al. (2010) Genetic control over the resting brain. Proc. Natl. Acad. Sci. U.S.A., 107, 1223-1228

19. Fornito, A., Zalesky, A., Bassett, D. S., Meunier, D., Ellison-Wright, I., Yücel, M., Wood, S. J., Shaw, K., O’Connor, J., Nertney, D., et al. (2011) Genetic influences on cost-efficient organization of human cortical functional networks. J. Neurosci., 31, 3261-3270

20. van den Heuvel, M. P., van Soelen, I. L. C., Stam, C. J., Kahn, R. S., Boomsma, D. I. and Hulshoff Pol, H. E. (2013) Genetic control of functional brain network efficiency in children. Eur Neuropsychopharmacol, 23, 19-23

21. Rubinov, M. and Sporns, O. (2010) Complex network measures of brain connectivity: uses and interpretations. Neuroimage, 52, 1059 1069

22. Glahn, D. C., Curran, J. E., Winkler, A. M., Carless, M. A., Kent, J. W. Jr, Charlesworth, J. C., Johnson, M. P., Göring, H. H., Cole, S. A., Dyer, T. D., et al. (2012) High dimensional endophenotype ranking in the search for major depression risk genes. Biol. Psychiatry, 71, 6-14

23. Frazer, K. A., Ballinger, D. G., Cox, D. R., Hinds, D. A., Stuve, L. L., Gibbs, R. A., Belmont, J. W., Boudreau, A., Hardenbol, P., Leal, S. M., et al., and the International HapMap Consortium. (2007) A second generation human haplotype map of over 3.1 million SNPs. Nature, 449, 851-861

24. Freedman, M. L., Reich, D., Penney, K. L., McDonald, G. J., Mignault, A. A., Patterson, N., Gabriel, S. B., Topol, E. J., Smoller, J. W., Pato, C. N., et al. (2004) Assessing the impact of population stratification on genetic association studies. Nat. Genet., 36, 388-393

25. Marchini, J., Cardon, L. R., Phillips, M. S. and Donnelly, P. (2004) The effects of human population structure on large genetic association studies. Nat. Genet., 36, 512-517

26. Devlin, B. and Roeder, K. (1999) Genomic control for association studies. Biometrics, 55, 997-1004

27. Price, A. L., Patterson, N. J., Plenge, R. M., Weinblatt, M. E., Shadick, N. A. and Reich, D. (2006) Principal components analysis corrects for stratification in genome-wide association studies. Nat. Genet., 38, 904-909

28. Kang, H. M., Sul, J. H., Service, S. K., Zaitlen, N. A., Kong, S. Y., Freimer, N. B., Sabatti, C. and Eskin, E. (2010) Variance component model to account for sample structure in genome-wide association studies. Nat. Genet., 42, 348-354

29. Nymberg, C., Jia, T., Lubbe, S., Ruggeri, B., Desrivieres, S., Barker, G., Büchel, C., Fauth-Buehler, M., Cattrell, A., Conrod, P., et al., and the IMAGEN Consortium. (2013) Neural mechanisms of attentiondeficit/hyperactivity disorder symptoms are stratified by MAOA 
genotype. Biol. Psychiatry, 74, 607-614

30. Joyner, A. H., J, C. R., Bloss, C. S., Bakken, T. E., Rimol, L. M., Melle, I., Agartz, I., Djurovic, S., Topol, E. J., Schork, N. J., et al. (2009) A common MECP2 haplotype associates with reduced cortical surface area in humans in two independent populations. Proc. Natl. Acad. Sci. U.S.A., 106, 15483-15488

31. Meyer-Lindenberg, A. (2009) Neural connectivity as an intermediate phenotype: brain networks under genetic control. Hum Brain Mapp, 30, 1938-1946

32. Brown, J. A., Terashima, K. H., Burggren, A. C., Ercoli, L. M., Miller, K. J., Small, G. W. and Bookheimer, S. Y. (2011) Brain network local interconnectivity loss in aging $A P O E-4$ allele carriers. Proc. Natl. Acad. Sci. U.S.A., 108, 20760-20765

33. Dennis, E. L., Jahanshad, N., Rudie, J. D., Brown, J. A., Johnson, K., McMahon, K. L., de Zubicaray, G. I., Montgomery, G., Martin, N. G., Wright, M. J., et al. (2011) Altered structural brain connectivity in healthy carriers of the autism risk gene, CNTNAP2. Brain Connect, 1 , 447-459

34. Loth, E., Poline, J. B., Thyreau, B., Jia, T., Tao, C., Lourdusamy, A., Stacey, D., Cattrell, A., Desrivières, S., Ruggeri, B., et al., and the IMAGEN Consortium. (2013) Oxytocin receptor genotype modulates ventral striatal activity to social cues and response to stressful life events. Biol. Psychiatry, PMID: 24120094

35. Friston, K. J., Ashburner, J. T., Kiebel, S. J., Nichols, T. E. and Penny, W. D. (2006) Statistical Parametric Mapping: The Analysis of Functional Brain Images. New York: Academic Press

36. Filippini, N., Rao, A., Wetten, S., Gibson, R. A., Borrie, M., Guzman, D., Kertesz, A., Loy-English, I., Williams, J., Nichols, T., et al. (2009) Anatomically-distinct genetic associations of $A P O E \& 4$ allele load with regional cortical atrophy in Alzheimer's disease. Neuroimage, 44, 724-728

37. Coon, K. D., Myers, A. J., Craig, D. W., Webster, J. A., Pearson, J. V., Lince, D. H., Zismann, V. L., Beach, T. G., Leung, D., Bryden, L., et al. (2007) A high-density whole-genome association study reveals that APOE is the major susceptibility gene for sporadic late-onset Alzheimer's disease. J Clin Psychiatry, 68, 613-618

38. Farrer, L. A., Cupples, L. A., Haines, J. L., Hyman, B., Kukull, W. A., Mayeux, R., Myers, R. H., Pericak-Vance, M. A., Risch, N. and van Duijn, C. M., and the APOE and Alzheimer Disease Meta Analysis Consortium. (1997) Effects of age, sex, and ethnicity on the association between apolipoprotein $\mathrm{E}$ genotype and Alzheimer disease. A meta-analysis. JAMA, 278, 1349-1356

39. Ho, A. J., Stein, J. L., Hua, X., Lee, S., Hibar, D. P., Leow, A. D., Dinov, I. D., Toga, A. W., Saykin, A. J., Shen, L., et al., and the Alzheimer's Disease Neuroimaging Initiative. (2010) A commonly carried allele of the obesity-related FTO gene is associated with reduced brain volume in the healthy elderly. Proc. Natl. Acad. Sci. U. S.A., 107, 8404-8409

40. Frayling, T. M., Timpson, N. J., Weedon, M. N., Zeggini, E., Freathy, R. M., Lindgren, C. M., Perry, J. R., Elliott, K. S., Lango, H., Rayner, N. W., et al. (2007) A common variant in the $F T O$ gene is associated with body mass index and predisposes to childhood and adult obesity. Science, 316, 889-894

41. Braskie, M. N., Jahanshad, N., Stein, J. L., Barysheva, M., McMahon, K. L., de Zubicaray, G. I., Martin, N. G., Wright, M. J., Ringman, J. M., Toga, A. W., et al. (2011) Common Alzheimer's disease risk variant within the $C L U$ gene affects white matter microstructure in young adults. J. Neurosci., 31, 6764-6770

42. Westlye, E. T., Lundervold, A., Rootwelt, H., Lundervold, A. J. and
Westlye, L. T. (2011) Increased hippocampal default mode synchronization during rest in middle-aged and elderly $A P O E \& 4$ carriers: relationships with memory performance. J. Neurosci., 31, 7775-7783

43. Filippini, N., MacIntosh, B. J., Hough, M. G., Goodwin, G. M., Frisoni, G. B., Smith, S. M., Matthews, P. M., Beckmann, C. F. and Mackay, C. E. (2009) Distinct patterns of brain activity in young carriers of the $A P O E-\varepsilon 4$ allele. Proc. Natl. Acad. Sci. U.S.A., 106, 7209-7214

44. Trachtenberg, A. J., Filippini, N., Ebmeier, K. P., Smith, S. M., Karpe, F. and Mackay, C. E. (2012) The effects of $A P O E$ on the functional architecture of the resting brain. Neuroimage, 59, 565-572

45. Chiang, M. C., Barysheva, M., Toga, A. W., Medland, S. E., Hansell, N. K., James, M. R., McMahon, K. L., de Zubicaray, G. I., Martin, N. G., Wright, M. J., et al. (2011) BDNF gene effects on brain circuitry replicated in 455 twins. Neuroimage, 55, 448-454

46. Thomason, M. E., Dougherty, R. F., Colich, N. L., Perry, L. M., Rykhlevskaia, E. I., Louro, H. M., Hallmayer, J. F., Waugh, C. E., Bammer, R., Glover, G. H., et al. (2010) COMT genotype affects prefrontal white matter pathways in children and adolescents. Neuroimage, 53, 926-934

47. Liu, B., Song, M., Li, J., Liu, Y., Li, K., Yu, C. and Jiang, T. (2010) Prefrontal-related functional connectivities within the default network are modulated by COMT val158met in healthy young adults. J. Neurosci., 30, 64-69

48. Scott-Van Zeeland, A. A., Abrahams, B. S., Alvarez-Retuerto, A. I., Sonnenblick, L. I., Rudie, J. D., Ghahremani, D., Mumford, J. A., Poldrack, R. A., Dapretto, M., Geschwind, D. H., et al. (2010) Altered functional connectivity in frontal lobe circuits is associated with variation in the autism risk gene CNTNAP2. Sci. Transl. Med., 2, $56 \mathrm{ra} 80$

49. Rudie, J. D., Hernandez, L. M., Brown, J. A., Beck-Pancer, D., Colich, N. L., Gorrindo, P., Thompson, P. M., Geschwind, D. H., Bookheimer, S. Y., Levitt, P., et al. (2012) Autism-associated promoter variant in MET impacts functional and structural brain networks. Neuron, 75 , 904-915

50. Braskie, M. N., Jahanshad, N., Toga, A. W., McMahon, K. L., de Zubicaray, G. I., Martin, N. G., Wright, M. J. and Thompson, P. M. (2012) How a common variant in the growth factor receptor gene, NTRK1, affects white matter. Bioarchitecture, 2, 181-184

51. Esslinger, C., Kirsch, P., Haddad, L., Mier, D., Sauer, C., Erk, S., Schnell, K., Amold, C., Witt, S. H., Rietschel, M., et al. (2011) Cognitive state and connectivity effects of the genome-wide significant psychosis variant in ZNF804A. Neuroimage, 54, 25142523

52. Johnson, A. D. and O'Donnell, C. J. (2009) An open access database of genome-wide association results. BMC Med. Genet., 10,6

53. Visscher, P. M., Brown, M. A., McCarthy, M. I. and Yang, J. (2012) Five years of GWAS discovery. Am. J. Hum. Genet., 90, 7-24

54. Potkin, S. G., Turner, J. A., Fallon, J. A., Lakatos, A., Keator, D. B., Guffanti, G. and Macciardi, F. (2009) Gene discovery through imaging genetics: identification of two novel genes associated with schizophrenia. Mol. Psychiatry, 14, 416-428

55. Stein, J. L., Hibar, D. P., Madsen, S. K., Khamis, M., McMahon, K. L., de Zubicaray, G. I., Hansell, N. K., Montgomery, G. W., Martin, N. G., Wright, M. J., et al., and the Alzheimer's Disease Neuroimaging Initiative Investigators. (2011) Discovery and replication of dopamine-related gene effects on caudate volume in young and elderly populations $(N=1198)$ using genome-wide search. Mol. Psychiatry, $16,927-937,881$ 
56. Potkin, S. G., Guffanti, G., Lakatos, A., Tumer, J. A., Kruggel, F., Fallon, J. H., Saykin, A. J., Orro, A., Lupoli, S., Salvi, E., et al., and the Alzheimer's Disease Neuroimaging Initiative. (2009) Hippocampal atrophy as a quantitative trait in a genome-wide association study identifying novel susceptibility genes for Alzheimer's disease. PLoS ONE, 4, e6501

57. Stein, J. L., Hua, X., Morra, J. H., Lee, S., Hibar, D. P., Ho, A. J., Leow, A. D., Toga, A. W., Sul, J. H., Kang, H. M., et al., and the Alzheimer's Disease Neuroimaging Initiative. (2010) Genome-wide analysis reveals novel genes influencing temporal lobe structure with relevance to neurodegeneration in Alzheimer's disease. Neuroimage, $51,542-554$

58. Chiang, M. C., Barysheva, M., McMahon, K. L., de Zubicaray, G. I., Johnson, K., Montgomery, G. W., Martin, N. G., Toga, A. W., Wright, M. J., Shapshak, P., et al. (2012) Gene network effects on brain microstructure and intellectual performance identified in 472 twins. J. Neurosci., 32, 8732-8745

59. Bralten, J., Arias-Vásquez, A., Makkinje, R., Veltman, J. A., Brunner, H. G., Fernández, G., Rijpkema, M. and Franke, B. (2011) Association of the Alzheimer's gene SORL1 with hippocampal volume in young, healthy adults. Am J Psychiatry, 168, 1083-1089

60. Shen, L., Kim, S., Risacher, S. L., Nho, K., Swaminathan, S., West, J. D., Foroud, T., Pankratz, N., Moore, J. H., Sloan, C. D., et al., and the Alzheimer's Disease Neuroimaging Initiative. (2010) Whole genome association study of brain-wide imaging phenotypes for identifying quantitative trait loci in $\mathrm{MCI}$ and $\mathrm{AD}$ : A study of the ADNI cohort. Neuroimage, 53, 1051-1063

61. Jahanshad, N., Rajagopalan, P., Hua, X., Hibar, D. P., Nir, T. M., Toga, A. W., Jack, C. R. Jr, Saykin, A. J., Green, R. C., Weiner, M. W., et al., and the Alzheimer's Disease Neuroimaging Initiative. (2013) Genome-wide scan of healthy human connectome discovers SPON1 gene variant influencing dementia severity. Proc. Natl. Acad. Sci. U.S. A., 110, 4768-4773

62. Stein, J. L., Hua, X., Lee, S., Ho, A. J., Leow, A. D., Toga, A. W., Saykin, A. J., Shen, L., Foroud, T., Pankratz, N., et al., and the Alzheimer's Disease Neuroimaging Initiative. (2010) Voxelwise genome-wide association study (vGWAS). Neuroimage, 53, 11601174

63. Leow, A., Huang, S. C., Geng, A., Becker, J. and Davis, S., et al. (2005) Inverse consistent mapping in 3D deformable image registration: its construction and statistical properties. In: Information Processing in Medical Imaging, pps, 493-503. Berlin: Springer

64. Hibar, D.P., Kohannim, O., Stein, J.L., Chiang, M. C. and Thompson. P.M. (2011) Multilocus genetic analysis of brain images. Frontiers in Genetics, 2, 73

65. Wu, M. C., Kraft, P., Epstein, M. P., Taylor, D. M., Chanock, S. J., Hunter, D. J. and Lin, X. (2010) Powerful SNP-set analysis for casecontrol genome-wide association studies. Am. J. Hum. Genet., 86, 929-942

66. Hoh, J., Wille, A. and Ott, J. (2001) Trimming, weighting, and grouping SNPs in human case-control association studies. Genome Res., 11, 2115-2119

67. Purcell, S., Neale, B., Todd-Brown, K., Thomas, L., Ferreira, M. A. R., Bender, D., Maller, J., Sklar, P., de Bakker, P. I., Daly, M. J., et al. (2007) PLINK: a tool set for whole-genome association and population-based linkage analyses. Am. J. Hum. Genet., 81, 559-575

68. Morgenthaler, S. and Thilly, W.G. (2007) A strategy to discover genes that carry multi-allelic or mono-allelic risk for common diseases: a cohort allelic sums test (CAST). Mutation Research/Fundamental and Molecular Mechanisms of Mutagenesis, 615, 28-56

69. Madsen, B. E. and Browning, S. R. (2009) A groupwise association test for rare mutations using a weighted sum statistic. PLoS Genet., 5, e1000384

70. Morris, A. P. and Zeggini, E. (2010) An evaluation of statistical approaches to rare variant analysis in genetic association studies. Genet. Epidemiol., 34, 188-193

71. Li, B. and Leal, S. M. (2008) Methods for detecting associations with rare variants for common diseases: application to analysis of sequence data. Am. J. Hum. Genet., 83, 311-321

72. Hoerl, R. W. (1985) Ridge analysis 25 years later. Am. Stat., 39, 186192.

73. Kohannim, O., Hibar, D. P., Stein, J. L., Jahanshad, N., Jack, C. R., Weiner, M. W., Toga, A. W. and Thompson, P. M. (2011) Boosting power to detect genetic associations in imaging using multi-locus, genome-wide scans and ridge regression. In Biomedical Imaging: From Nano to Macro, 2011 IEEE International Symposium, IEEE, 1855-1859

74. Tibshirani, R. (1996) Regression shrinkage and selection via the lasso. J. R. Stat. Soc. Series B Stat. Methodol., 58, 267-288.

75. Kohannim, O., Hibar, D.P., Stein, J.L., Jahanshad, N., Hua, X., et al. (2012) Discovery and replication of gene influences on brain structure using LASSO regression. Frontiers in Neuroscience, 6, 115

76. Zou, H. and Hastie, T. (2005) Regularization and variable selection via the elastic net. J. R. Stat. Soc. Series B Stat. Methodol., 67, 301-320.

77. Kohannim, O., Hibar, D. P., Jahanshad, N., Stein, J. L., Hua, X., Toga, A. W., Jack, C. R., Weinen, M. W. and Thompson, P. M. (2012) Predicting temporal lobe volume on MRI from genotypes using $1^{1}-1^{2}$ regularized regression. In Biomedical Imaging: From Nano to Macro, 2012 IEEE International Symposium, IEEE, 1160-1163.

78. Hibar, D. P., Stein, J. L., Kohannim, O., Jahanshad, N., Saykin, A. J., Shen, L., Kim, S., Pankratz, N., Foroud, T., Huentelman, M. J., et al., and the Alzheimer's Disease Neuroimaging Initiative. (2011) Voxelwise gene-wide association study (vGeneWAS): multivariate genebased association testing in 731 elderly subjects. Neuroimage, 56, 1875-1891

79. Ge, T., Feng, J., Hibar, D. P., Thompson, P. M. and Nichols, T. E. (2012) Increasing power for voxel-wise genome-wide association studies: the random field theory, least square kernel machines and fast permutation procedures. Neuroimage, $63,858-873$

80. Liu, D., Lin, X. and Ghosh, D. (2007) Semiparametric regression of multidimensional genetic pathway data: least-squares kernel machines and linear mixed models. Biometrics, 63, 1079-1088

81. Wu, M. C., Lee, S., Cai, T., Li, Y., Boehnke, M. and Lin, X. (2011) Rare-variant association testing for sequencing data with the sequence kernel association test. Am. J. Hum. Genet., 89, 82-93

82. Kwee, L. C., Liu, D., Lin, X., Ghosh, D. and Epstein, M. P. (2008) A powerful and flexible multilocus association test for quantitative traits. Am. J. Hum. Genet., 82, 386-397

83. Lin, X. (1997) Variance component testing in generalised linear models with random effects. Biometrika, 84, 309-326.

84. Hotelling, H. (1936) Relations between two sets of variates. Biometrika, 28, 321-377

85. Wold, S., Martens, H. and Wold, H. (1983) The multivariate calibration problem in chemistry solved by the PLS method. Matrix Pencils, 973, 286-293

86. Le Floch, E., Guillemot, V., Frouin, V., Pinel, P., Lalanne, C., 
Trinchera, L., Tenenhaus, A., Moreno, A., Zilbovicius, M., Bourgeron, T., et al. (2012) Significant correlation between a set of genetic polymorphisms and a functional brain network revealed by feature selection and sparse Partial Least Squares. Neuroimage, 63, 11-24

87. Chi, E. C., Allen, G. I., Zhou, H., Kohannim, O., Lange, K., et al. (2013) Imaging genetics via sparse canonical correlation analysis. In: Biomedical Imaging: From Nano to Macro, 2013 IEEE International Symposium, IEEE, 740-743

88. Liu, J., Pearlson, G., Windemuth, A., Ruano, G., Perrone-Bizzozero, N. I. and Calhoun, V. (2009) Combining fMRI and SNP data to investigate connections between brain function and genetics using parallel ICA. Hum Brain Mapp, 30, 241-255

89. Wang, H., Nie, F., Huang, H., Kim, S., Nho, K., Risacher, S. L., Saykin, A. J. and Shen, L., and the Alzheimer's Disease Neuroimaging Initiative. (2012) Identifying quantitative trait loci via groupsparse multitask regression and feature selection: an imaging genetics study of the ADNI cohort. Bioinformatics, 28, 229-237

90. Wang, H., Nie, F., Huang, H., Risacher, S. L., Saykin, A. J. and Shen, L., and the Alzheimer's Disease Neuroimaging Initiative. (2012) Identifying disease sensitive and quantitative trait-relevant biomarkers from multidimensional heterogeneous imaging genetics data via sparse multimodal multitask learning. Bioinformatics, 28, i127-i136

91. Vounou, M., Nichols, T. E. and Montana, G., and the Alzheimer's Disease Neuroimaging Initiative. (2010) Discovering genetic associations with high-dimensional neuroimaging phenotypes: A sparse reduced-rank regression approach. Neuroimage, 53, 1147-1159

92. Vounou, M., Janousova, E., Wolz, R., Stein, J. L., Thompson, P. M., Rueckert, D. and Montana, G., and the Alzheimer's Disease Neuroimaging Initiative. (2012) Sparse reduced-rank regression detects genetic associations with voxel-wise longitudinal phenotypes in Alzheimer's disease. Neuroimage, 60, 700-716

93. Silver, M., Janousova, E., Hua, X., Thompson, P. M. and Montana, G., and the Alzheimer's Disease Neuroimaging Initiative. (2012) Identification of gene pathways implicated in Alzheimer's disease using longitudinal imaging phenotypes with sparse regression. Neuroimage, 63, 1681-1694

94. Stingo, F. C., Guindani, M., Vannucci, M. and Calhoun, V. D. (2013) An integrative Bayesian modeling approach to imaging genetics. J. Am. Stat. Assoc., 108, 876-891

95. Batmanghelich, N. K., Dalca, A. V., Sabuncu, M. R. and Golland, P. (2013) Joint modeling of imaging and genetics. In Information Processing in Medical Imaging, Berlin: Springer, pps 766-777

96. Guyon, I., Weston, J., Barnhill, S. and Vapnik, V. (2002) Gene selection for cancer classification using support vector machines. Mach. Learn., 46, 389-422

97. Zhang, H. H. and Lv, J. (2008) Discussion of "Sure Independence Screening for Ultra-High Dimensional Feature Space”. J. R. Stat. Soc. Series B Stat. Methodol., 70, 849-911

98. Fan, J. and Song, R. (2010) Sure independence screening in generalized linear models with NP-dimensionality. Ann. Stat., 38, 3567-3604.

99. He, Q. and Lin, D. Y. (2011) A variable selection method for genomewide association studies. Bioinformatics, 27, 1-8

100. Benjamini, Y. and Hochberg, Y. (1995) Controlling the false discovery rate: a practical and powerful approach to multiple testing. J. R. Stat. Soc. Series B Stat. Methodol., 57, 289-300.

101. Genovese, C. R., Lazar, N. A. and Nichols, T. (2002) Thresholding of statistical maps in functional neuroimaging using the false discovery rate. Neuroimage, $15,870-878$

102. Holmes, A. P., Blair, R. C., Watson, J. D. and Ford, I. (1996) Nonparametric analysis of statistic images from functional mapping experiments. J. Cereb. Blood Flow Metab., 16, 7-22

103. Nichols, T. and Hayasaka, S. (2003) Controlling the familywise error rate in functional neuroimaging: a comparative review. Stat Methods Med Res, 12, 419-446

104. Knijnenburg, T. A., Wessels, L. F. A., Reinders, M. J. T. and Shmulevich, I. (2009) Fewer permutations, more accurate $P$-values. Bioinformatics, 25, i161-i168

105. Friston, K. J., Holmes, A., Poline, J. B., Price, C. J. and Frith, C. D. (1996) Detecting activations in PET and fMRI: levels of inference and power. Neuroimage, 4, 223-235

106. Worsley, K. J., Marrett, S., Neelin, P., Vandal, A. C., Friston, K. J. and Evans, A. C. (1996) A unified statistical approach for determining significant signals in images of cerebral activation. Hum Brain Mapp, $4,58-73$

107. Cao, J. (1999) The size of the connected components of excursion sets of $\chi^{2}, \mathrm{~T}$ and F fields. Adv. Appl. Probab., 31, 579-595.

108. Smith, S. M. and Nichols, T. E. (2009) Threshold-free cluster enhancement: addressing problems of smoothing, threshold dependence and localisation in cluster inference. Neuroimage, 44, 83-98

109. Zhang, H., Nichols, T. E. and Johnson, T. D. (2009) Cluster mass inference via random field theory. Neuroimage, 44, 51-61

110. Chumbley, J., Worsley, K., Flandin, G. and Friston, K. (2010) Topological FDR for neuroimaging. Neuroimage, 49, 3057-3064

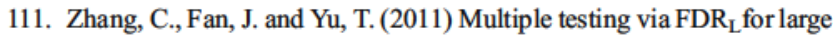
scale imaging data. Ann Stat, 39, 613-642

112. Gao, X., Starmer, J. and Martin, E. R. (2008) A multiple testing correction method for genetic association studies using correlated single nucleotide polymorphisms. Genet. Epidemiol., 32, 361-369

113. Gao, X., Becker, L. C., Becker, D. M., Starmer, J. D. and Province, M. A. (2010) Avoiding the high Bonferroni penalty in genome-wide association studies. Genet. Epidemiol., 34, 100-105

114. Fan, J., Han, X. and Gu, W. (2012) Estimating false discovery proportion under arbitrary covariance dependence. J. Am. Stat. Assoc., $107,1019-1035$.

115. Efron, B. (2013) Large-scale Inference: Empirical Bayes Methods for Estimation, Testing, and Prediction. Cambridge: Cambridge University Press

116. Eklund, A., Andersson, M.and Knutsson. H. (2011) Fast random permutation tests enable objective evaluation of methods for singlesubject fMRI analysis. International Journal of Biomedical Imaging. http://dx.doi.org/10.1155/2011/627947

117. Nichols, T. E. (2012) Multiple testing corrections, nonparametric methods, and random field theory. Neuroimage, 62, 811-815

118. Ioannidis, J. P. A. (2005) Why most published research findings are false. PLoS Med., 2, e124

119. Sullivan, P. F. (2010) The psychiatric GWAS consortium: big science comes to psychiatry. Neuron, $68,182-186$

120. Schumann, G., Loth, E., Banaschewski, T., Barbot, A., Barker, G., Büchel, C., Conrod, P. J., Dalley, J. W., Flor, H., Gallinat, J., et al., and the IMAGEN consortium. (2010) The IMAGEN study: reinforcement-related behaviour in normal brain function and psychopathology. Mol. Psychiatry, 15, 1128-1139

121. Stein, J. L., Medland, S. E., Vasquez, A. A., Hibar, D. P., Senstad, R. E., Winkler, A. M., Toro, R., Appel, K., Bartecek, R., Bergmann, Ø., et al., and the Alzheimer's Disease Neuroimaging Initiative, and the 
EPIGEN Consortium, and the IMAGEN Consortium, and the Saguenay Youth Study Group, and the Cohorts for Heart and Aging Research in Genomic Epidemiology Consortium, and the Enhancing Neuro Imaging Genetics through Meta-Analysis Consortium. (2012) Identification of common variants associated with human hippocampal and intracranial volumes. Nat. Genet., 44, 552-561

122. Jahanshad, N., Kochunov, P. V., Sprooten, E., Mandl, R. C., Nichols, T. E., Almasy, L., Blangero, J., Brouwer, R. M., Curran, J. E., de
Zubicaray, G. I., et al. (2013) Multi-site genetic analysis of diffusion images and voxelwise heritability analysis: a pilot project of the ENIGMA-DTI working group. Neuroimage, 81, 455-469

123. Novak, N. M., Stein, J. L., Medland, S. E., Hibar, D. P., Thompson, P. M. and Toga, A. W. (2012) EnigmaVis: online interactive visualization of genome-wide association studies of the Enhancing NeuroImaging Genetics through Meta-Analysis (ENIGMA) consortium. Twin Res. Hum. Genet., 15, 414-418 